\title{
Cross-cultural invariance of NPI-13: Entitlement as culturally specific, leadership and grandiosity as culturally universal
}

\begin{abstract}
The current study explores the problem with the lack of measurement invariance for the Narcissistic Personality Inventory (NPI) by addressing two issues: conceptual heterogeneity of narcissism and methodological issues related to the binary character of data. We examine the measurement invariance of the 13-item version of the NPI in three populations in Japan, Poland and the UK. Analyses revealed that leadership/authority and grandiose exhibitionism dimensions of the NPI were cross-culturally invariant, while entitlement/exploitativeness was culturally specific. Therefore, we proposed NPI-9 as indicating scalar invariance, and we examined the pattern of correlations between NPI-9 and other variables across three countries. The results suggest that NPI-9 is valid brief scale measuring general levels of narcissism in cross-cultural studies, while the NPI-13 remains suitable for research within specific countries.
\end{abstract}

Keywords: NPI; narcissism; measurement invariance; validity; cross-cultural comparisons 
CROSS-CULTURAL GENERALISABITY OF THE NPI

Cross-cultural studies of narcissism have increased in recent years (Brailovskaia \& Bierhoff, 2016; Cozma, Javadian, Gupta, \& Canever, 2014; Meisel, Ning, Campbell, \& Goodie, 2016). However, numerous researchers report problems with measurement invariance of the Narcissistic Personality Inventory (NPI; e.g. Cozma et al., 2014; Meisel et al., 2016). In the current paper we examine to what extent cross-cultural comparisons are justified based on scoring of the abbreviated version of the Narcissistic Personality Inventory (the NPI-13;

Gentile et al., 2013) in three countries: Japan, Poland, and United Kingdom. We propose more in-depth analyses of factor structure of NPI following Ackerman et al. (2011) model and we use tetrachoric correlations instead of commonly used Person's correlation matrix respecting binary character of data obtained using the NPI.

\section{The concept and measurement of narcissism}

The concept of narcissism is one of the most popular constructs in personality and social psychology (Miller \& Campbell, 2008). It is typically defined as a grandiose self-view, a need for power and feelings of entitlement (see Miller \& Campbell, 2008 for a review). Undoubtedly the most popular scale for measuring narcissism is the Narcissistic Personality Inventory (NPI; Raskin \& Terry, 1988). The original scale is comprised of 40 items. A threefactor solution for the NPI-40 was originally proposed (Ackerman et al., 2011) comprising leadership/authority (LA), grandiose exhibitionism (GE), and entitlement/exploitativeness (EE). Both, LA and GE are related to socially potential positive outcomes such as extraversion, goal-persistence, and self-esteem, while EE is related to socially toxic negative outcomes such as low empathy, low self-esteem, aggressiveness, neuroticism, disagreeableness, Machiavellianism, and antisocial tendencies (Ackerman et al., 2011; Gentile et al., 2013).

Two abbreviated versions of the NPI-40 were proposed: the NPI-16 (Ames, Rose, \& Anderson, 2006) and the NPI-13 (Gentile et al., 2013). Whereas the NPI-16 is suited for the 
CROSS-CULTURAL GENERALISABITY OF THE NPI

studies that focus on assessing the global construct of narcissism, the NPI-13 has been validated to assess the three subscales described by Ackerman et al. (2011). The items in NPI16 (Ames, et al., 2006) were chosen based on two criteria: 1) face validity and 2) coverage of different dimensions distinguished in literature. Ames et al. (2006) argued that a shortened to less than 20 items measure of NPI is needed, but also that should be longer than 10 items for the sake of reliability, which resulted in 16 pair of items. These items were further tested and resulted to be externally valid and stable over time (Ames et al., 2006). The weakness of the NPI-16 is the fact that during the construction study (Ames et al., 2006) its structure was not verified. Gentile et al. (2013) during the construction of the NPI-13 compared its structure with the NPI-16 (Ames et al., 2006) and demonstrated that the NPI-16 measures not one global narcissism but five distinct factors. The NPI-13 (Gentile et al., 2013) used the factorial analytic work by Ackerman et al. (2011) on the full-length 40 item version and constructed its shortened version but measuring the very same factors (i.e., the EE, LA and GE), and it has already been successfully used in other cultures demonstrating very good psychometric properties (e.g., Brailovskaia, Bierhoff, \& Margraf, 2017).

\section{Measurement invariance of NPI}

Despite increasing interest in the cross-cultural studies on narcissism, there are few studies examining the measurement invariance of the NPI (see Cozma et al., 2014 for a review). Measurement invariance is crucial in cross-cultural studies, as it allows for the assessment of the extent to which any cross-cultural comparisons are justified. Generally, there are three basic levels of measurement invariance: configural - which answers the question whether the factor structure of scales is similar across compared groups; metric which answers the question of whether the factor loading of items are similar across groups, allowing the comparison of predictors or correlates of a construct across groups; and scalar which answers the question of whether item intercepts are equal intercepts across groups 
CROSS-CULTURAL GENERALISABITY OF THE NPI

(Bieda et al., 2016; Meredith, 1993). Metric level of invariance is not assessed with binary variables (such as from the NPI) due to scale factors or residual variances being allowed to vary across groups (Muthén \& Muthén, 2014), and it is recommended to go straight from configural to scalar invariance (Muthén \& Muthén, 2014).

Cozma et al. (2014) in their review on former cross-cultural studies of the NPI did not find any paper reporting higher than configural invariance for the scale. More recently, Meisel et al. (2016) also did not establish configural invariance for NPI-40 in U.S. and Chinese samples. However, it is worth noting that both Cozma et al. (2014) and Meisel et al. (2016) used the maximum likelihood estimation method and therefore tried to assess metric invariance, which is inappropriate for the data gathered by NPI. Although Cozma et al. (2014) reported metric invariance for the NPI-16 scale (Ames et al., 2006), they were able to do so due to correlating multiple error variances in the model, while initially the fit indices of the measurement model were suggesting poor fit to the data (which suggests no configural invariance at all).

The current study

The main aim of the current study is to examine whether narcissism, as measured by the NPI, can be measured across diverse cultures. The concept of narcissism was developed in a Western culture, and the scale measuring narcissism was validated in English-speaking populations. Thus, testing the measurement invariance of the NPI across cultures should include populations from outside of English-speaking countries. For this reason, we examine the measurement invariance of the NPI in three different countries: Japan, Poland, and the United Kingdom, and its applicability in cross-cultural studies.

We chose these countries because Poland is a Central-European country with a different cultural background to that of the United Kingdom and Japan is unique in terms of its values, religion and language (Hofstede et al., 2010). All these countries differ in their 
CROSS-CULTURAL GENERALISABITY OF THE NPI

individualism levels, where the UK is an individualistic country, Japan collectivistic, and Poland is placed in the middle of the individualism-collectivism dimension (Hofstede et al., 2010). Lower levels of narcissism have been reported among collectivistic or Eastern samples than among individualistic or Western samples, e.g., Fukunishi et al. (1996) reported that the Japanese indicated a lower level of narcissism in comparison to Americans and Chinese.

Despite China and Japan have treated as both collectivistic countries, they significantly differ in their orientations toward interdependence. For instance, Japanese conception of happiness (Hitokoto \& Uchida, 2015), involves being ordinary, i.e., oriented towards being similar to ingroup others and building personal satisfaction in harmony with others. Being narcissistic in such a context undermine rejection by others, therefore collectivism in Japan is marked by its strong rejection avoidance, which can be measured by self-report scale (Hashimoto \& Yamagishi, 2013; Noguchi, 2007). In contrast, the Chinese self involves duality of selfenhancement that manifest in implicit self-effacement in their in-group and explicit selfenhancement (Yamaguchi et al., 2007). To elucidate the largest cultural variation relevant for the current self-enhancement study using self-report, we considered best to include Japan as one representative of collectivistic cultures. Thus, we expect that Japan will score lowest on narcissism as in comparison to Poland and the United Kingdom.

We chose the NPI-13 for examining measurement invariance as this version allows for identifying the three scales assumed by the Ackerman et al (2011) model. Two main sources of lack of measurement invariance are assumed: (1) the heterogeneous nature of grandiose narcissism, as indicated by Ackerman et al. (2011); and (2) measurement issues, related to ignoring the binary character of data.

We supplement our analyses by examining the pattern of correlations between narcissism and other variables: self-esteem, psychological entitlement, communal narcissism, interdependent self-construal, and interdependent happiness across three countries. Positive 
CROSS-CULTURAL GENERALISABITY OF THE NPI

relationships between narcissism, positive self-esteem, and psychological entitlement are reported in numerous studies (e.g., Campbell, Rudich, \& Sedikides, 2002). Communal narcissism is assumed to be a parallel form of agentic grandiose narcissism (Gebauer et al., 2012). Finally, interdependent self-construal is logically opposite to narcissism, as it assumes focus on interpersonal relationships and defining self in terms of close relationships with others (Singelis, 1994), while interdependent happiness is related to the Japanese lay conception of happiness (Hitokoto \& Uchida, 2015; Uchida \& Kitayama, 2009).

In accordance to previous studies (Fukunishi et al., 1995), we would expect that Japanese should score lower in narcissism than European countries, and in accordance to the results comparing similar populations (Brailovskaia \& Bierhoff, 2016), we expect no differences between Poland and the UK. However, as no study has demonstrated measurement invariance around the NPI (Cozma et al., 2014), we also hypothesize that this measurement invariance will not occur. However, we also aim to investigate this issue and detect the cause of this variance. In regard to the correlates, we expect positive correlations between narcissism, self-esteem, psychological entitlement and communal narcissism and negative (or non-significant) correlations of narcissism to interdependent self and interdependent happiness across the three countries.

\section{Methods}

\section{$\underline{\text { Participants and procedure }}$}

Data were collected from university students in three countries: Japan $(N=179 ; 90$ women, 87 men, 2 people did not answer the question about their sex $)$; Poland ( $N=298 ; 172$ women, 126 men); the UK $(N=187 ; 141$ women, 46 men). The age range of the Japanese sample was from 18 to 27 years $(M=20.31, S D=1.23)$, the Polish sample from 18 to 25 years $(M=21.98$, $S D=1.60)$, and the UK sample from 18 to 30 years $(M=19.44, S D=2.06)$. Students majored 
CROSS-CULTURAL GENERALISABITY OF THE NPI

in social sciences and humanities field (mostly, but not limited to psychology). Informed consent was obtained from all participants.

In all countries, the national versions of the scales were used (after a back translation procedure; Brislin, 1986), that is, Japanese in Japan, Polish in Poland, and English in the UK. To examine the validity of translated versions, we correlated NPI with psychological entitlement (Campbell et al, 2002), self-esteem (Rosenberg, 1965) and communal narcissism (Gebauer et al., 2012), as these variables systematically correlated to narcissism in former studies. Additionally, the relational-interdependent self-construal (Cross, Bacon, \& Morris, 2000) and interdependent happiness (Hitokoto \& Uchida, 2015) were included as logically independent with narcissism.

Data from the Polish and Japanese samples were collected by the Ariadna data collection system, and from the English sample via the university online research system. Participants were rewarded by course credit points. Prior to data collection, participants were informed about anonymity and voluntary participation; after data collection, they were informed about the purpose of the study.

\section{$\underline{\text { Measures }}$}

NPI-13 (Gentile et al., 2013). It is an abbreviated form of the full version of NPI-40 (Raskin \& Terry, 1988), used in non-clinical populations and validated in numerous studies. The scale is comprised of 13 items (e.g. "I like having authority over other people versus I don't mind following orders") with a binary system of answers $(0=$ low narcissism, $1=$ high narcissism).

Rosenberg Self-esteem Scale (RSES, Rosenberg, 1965). The SES is a measure of general positive self-evaluation. It consists of 10 items (e.g. "On the whole, I am satisfied with myself'), five are reversely scored. Participants answer the questions on a five-point Likertlike scale from $1=$ I strongly disagree to $5=$ I strongly agree. 
CROSS-CULTURAL GENERALISABITY OF THE NPI

Psychological entitlement scale (PES, Campbell, Bonacci, Shelton, Exline, \& Bushman, 2004). The PES measures psychological entitlement, i.e. belief that the individual deserves more than others and is entitled to more than them. The scale consists of nine items (e.g. "I deserve the best"), one is reversely scored. Participants answer on a seven-point scale from $1=\mathrm{I}$ strongly disagree to $7=\mathrm{I}$ strongly agree .

Communal Narcissism Inventory (CNI, Gebauer et al., 2012). The scale measures communal narcissism, i.e. grandiose self-view in the communal domain (e.g. " I'm great listener", "I will bring the freedom to all humanity"). The scale consists of 16 items, with a seven-point Likert-like system answering from $1=\mathrm{I}$ strongly disagree to $7=$ I strongly agree.

Interdependent happiness scale (IHS, Hitokoto \& Uchida, 2015). This scale measures concept of interdependent happiness. It consists of nine items (e.g. "Although it is quite average, I live a stable life"), each of them with answers ranging from $1=\mathrm{I}$ strongly disagree to $7=$ I strongly agree.

Relational-independent self-construal scale (RISC, Cross et al., 2000). The scale serves as a measure of relational self-construal, the highest result denotes higher interdependency levels (Cross et al., 2000). It contains 11 items (e.g. “My close relationships are an important reflection of who I am"). The answer categories range from 1 = I strongly disagree to $5=$ I strongly agree.

\section{$\underline{\text { Statistical analyses }}$}

Because the response scale of the NPI-13 comprises of just two response categories, standard estimation methods were not suitable. Therefore, in all of the tested models we used the tetrachoric correlation matrix with the WLSMV estimator. Confirmatory factor and measurement invariance analyses were carried out using Mplus v. 7.2 (Muthén \& Muthén, 2014). In the assessment of measurement invariance model fit we followed Chen (2007) recommendations for small groups with different sample size, i.e., the difference between 
CROSS-CULTURAL GENERALISABITY OF THE NPI

compared adjacent models should not exceed .005 in CFI and .010 in RMSEA. However,

Chen (2007) developed these recommendations for the maximum likelihood estimator, whereas in current study our data has the binary character we treated these recommendations serve rather as a guide in interpretation than arbitrary golden rules. We assume that the results are invariant when the difference between configural and scalar level should not exceed .010 in CFI and .020 in RMSEA (as the metric model is omitted in the analyses).

\section{Results}

As a preliminary step, we tested for the measurement invariance of the RISC scale, as it is often additionally used as a manipulation check of whether the cultural groups differ in their self-concept (Meisel et al., 2016). Based on robust maximum likelihood estimation, both configural $\left(\chi^{2}=141.03_{(60)} ; p<.001 ; \mathrm{CFI}=.935 ; \mathrm{RMSEA}=.078 ; \mathrm{SRMR}=.047\right)$ and metric $\left(\chi^{2}=161.72_{(74)} ; p<.001 ; \mathrm{CFI}=.929 ; \mathrm{RMSEA}=.073 ; \mathrm{SRMR}=.077\right)$ models were well fitted to the data, whereas the scalar model was poorly fitted to the data $\left(\chi^{2}=243.73_{(88)} ; p<.001\right.$; $\mathrm{CFI}=.874 ; \mathrm{RMSEA}=.089 ; \mathrm{SRMR}=.097)$. According to Chen's $(2007)$ recommendations, compared models are invariant on a metric level because the difference in the approximate fit indices of the configural and metric model was in acceptable range $(\Delta \mathrm{CFI}=.006 ; \Delta \mathrm{RMSEA}$ $=.005 ; \Delta \mathrm{SRMR}=.030)$, therefore it is possible to compare relationships with other constructs between samples. Because the difference in fit indices between metric and scalar level exceeded assumed acceptable range (Chen, 2007) it turned out that it is impossible to check whether the levels of interdependent self-construal vary as a function of nationality.

The descriptive statistics and reliability estimates of applied measures for each country are presented in Table 1.

Before the assessment of measurement invariance of narcissism across Japan, Poland, and United Kingdom, the three-factor model was examined in terms of goodness-of-fit to the 
CROSS-CULTURAL GENERALISABITY OF THE NPI

data in each country separately. A summary of fit indices of those models is presented in Table 2.

The three factor model was well fitted to the data in each country. The multi-group confirmatory analyses were conducted to examine the measurement invariance for NPI-13. Compared groups were invariant only on the configural level $\left(\chi^{2}=257.72_{(186)} ; p<.001 ; \mathrm{CFI}=\right.$ $.941 ;$ RMSEA $=.042$ [.028-.053]; $p=.871 ;$ the scalar model did not converge), which confirmed that generally narcissism is similarly conceptualised in each country. However, the lack of scalar invariance suggests that ethnic group influenced latent scores. To explore the potential source of lack of invariance, we descended in our analyses to the item-level. The differences in answers distribution across the three countries were examined using the $\chi^{2}$ test and the results are presented in Table 3.

The differences between answers' distribution in ten items were non-significant in at least one dyad. The differences in answers distribution were significant for three items $(4,7$, and 10) in all compared dyads and all of them comprised the EE scale. Despite the fact that the distribution of answers of the last remaining item from the EE scale (i.e. "I find it easy to manipulate people") was non-significant in all compared dyads, it was excluded from further analyses to maintain construct validity. Therefore, a two-factor model of narcissism was examined in multi-group confirmatory factor analysis. Results of measurement invariance analysis are presented in Table 4.

The examined model was well fitted to the data on both configural and scalar level. Although $\triangle$ CFI slightly exceeded Chen's (2007) recommendations, $\triangle$ RMSEA suggests that compared populations are invariant.

After establishing the scalar invariance, we compared latent means of LA and GE across Polish, Japanese, and British populations, which results are presented in Table 5. No significant differences were found between latent means of Japan, Poland, and the United 
CROSS-CULTURAL GENERALISABITY OF THE NPI

Kingdom. On the one hand, the difference in GE between Poland and other compared populations was close to zero, whereas on the other - the difference in LA between Poland and other compared populations was highest, though still non-significant. The Japanese population scored relatively low in both GE and LA, with the exception of the latent mean score in LA with British populations, which was close to zero.

The abbreviated version of NPI seems to represent a narrower concept than the original one (i.e. excluding EE), so one could doubt if the cross-culturally invariant NPI-9 represents a measure of narcissism reasonably similar to NPI-13. Therefore, correlations between NPI-13, NPI-9, psychological entitlement, self-esteem, communal narcissisms, interdependent self and interdependent happiness in three countries were examined. These correlations are reported in Table 6.

Results confirmed the theoretical validity of NPI-9. There were significant differences between the strength of correlations with external variables between NPI-13 and NPI-9, however they were not substantial as the mean correlation strength across compared variables equaled only .05 . Moreover, the correlation between NPI-13 and NPI-9 was very high $(r=$ $.92 ; p<.001)$. Thus, our results suggest that it is plausible to reduce the NPI-13 to NPI-9 in cross-cultural research, as it demonstrates scalar invariance and maintain stable correlational pattern.

\section{Discussion}

Despite the NPI is one of the most widely used instruments measuring narcissism (Raskin \& Terry, 1988), no studies reported scalar invariance for cross-cultural comparisons. Given the repeated problems with established measurement invariance for NPI (Cozma et al., 2014; Meisel et al., 2016), two solutions for these problems were proposed: (i) examination of the cultural universality versus specificity of three dimensions of narcissism; (ii) examine measurement invariance by using a tetrachoric correlation matrix, appropriate to the binary 
CROSS-CULTURAL GENERALISABITY OF THE NPI

character of data. We chose NPI-13 for examining the narcissism concept as this scale reflects three basic components of narcissism: leadership/authority (LA), grandiose exhibitionism (GE) and entitlement/exploitativeness (EE).

Based on results of measurement invariance analysis of the NPI-13, we could claim that, in all three countries, the three-factor concept of narcissism (Ackerman et al., 2011) is similar; however, the lack of scalar invariance suggests that it is impossible to compare predictors of three-factor narcissism and its latent mean scores across cultures.

We identified that only distribution of answers on items from the EE significantly differed in all compared dyads. LA and GE scales form a two-factor scale appropriate to cross-cultural comparisons, allowing examination of both correlates/predictors of narcissism and reliable comparisons in the narcissism level across countries. The results of cross-country comparisons in the levels of narcissism were congruent with former studies (Fukunishi et al., 1996). We have found that although the Japanese tend to score relatively lower in narcissism levels than the Polish and the British, the difference is not significant. The lack of observed differences across different cultures was also found in previous studies, e.g., between Germany and Russia (Brailovskaia \& Bierhoff, 2016).

As our analyses suggest that only the LA-GE part of the narcissism model is crossculturally replicable in terms of scalar invariance, we examined whether this narrower scale still reflects the narcissism concept in a sufficient way. Therefore, we correlated NPI-9 with external variables in the three compared countries. We expected a similar pattern of correlates for NPI-13 and NPI-9 and a replicable pattern of correlations across countries: positive correlations with self-esteem, psychological entitlement and communal narcissism, and negative or lack of correlations with interdependent happiness and interdependent self. We demonstrated that NPI-13 and NPI-9 correlated in a similar manner to external variables. As in comparison to the NPI-40, the NPI-9 as well as the NPI-13 (Gentile et al., 2013) is just its 
CROSS-CULTURAL GENERALISABITY OF THE NPI

shortened version with the same hypothesized factorial structure but the difference lies in the exclusion of the EE factor, which turned out to be difficult in cross-cultural comparisons.

Therefore, we could claim that despite the NPI-9 measuring a narrower concept of narcissism than the NPI-13, it is still a good and valid measure of grandiose narcissism. Its biggest advantage over other existing version of the NPI is the measurement invariance across three diverse cultures, which proves its usefulness in cross-cultural studies. However, if one is interested in examining narcissism in one specific culture, the NPI-13 could be used for research within specific countries, as it covers all traditional aspects of narcissism, but when comparisons of narcissism are needed to be made across countries, NPI-9 would be most suitable to achieve this aim.

\section{Limitations and further directions}

This study has its limitations, such as the young age of the participants, the selection of cultures, and the chosen scale. As our study was conducted on student samples, it has rather a preliminary character - and so to be more confident about our proposition, the replication of the obtained results in other cultures and on non-student samples is needed. Also, in the UK sample there were significantly more women than men, even in comparison to two other samples. Meta-analyses on gender differences in narcissism suggests that men score higher than women (Grijalva et al., 2015), and therefore given the disproportionate number of women in the UK sample, this might have additionally influenced our findings.

\section{Summary}

Our study offers the very first proposal of a valid and short version of the NPI which is appropriate in cross-cultural comparisons. Therefore, it could be useful in further exploration of cross-cultural differences in the narcissism levels and predictors or correlates of this phenomenon. Our study pointed that EE is this aspect of narcissism which is understood in different ways across compared countries. However, excluding EE from the universal model 
CROSS-CULTURAL GENERALISABITY OF THE NPI

is too simple a solution of the problem. Another remedy would be re-thinking about the narcissism construct universe by using multi-cultural perspectives, and how to establish such a bottom-up item pool. Moreover, the interplay between cultural context and narcissism would need to employ more comprehensive, cross-cultural studies, using multi-level analysis. Our results have rather preliminary character and may serve as a guide for future research on cross-cultural differences in narcissism suggesting that with current state of art measurement of maladaptive expressions of narcissism is troublesome., It might be promising to test the cultural differences in narcissism using NPI with the Likert-type scale which already demonstrated its utility (Ackerman, Donnellan, Roberts, \& Fraley, 2016). Such procedure might help us to better understand narcissism in cross-cultural context, however more research, possibly including more countries is needed. 
CROSS-CULTURAL GENERALISABITY OF THE NPI

\section{References}

Ackerman, R.A., Donnellan, M.B., Roberts, B.W., \& Fraley, R.Ch. (2016). The effect of response format on the psychometric properties of the Narcissistic Personality Inventory. Assessment, 23, 203-220. doi:10.1177/1073191114568113

Ackerman, R.A., Witt, E.A., Donnellan, M.B., Trzesniewski, K.H., Robins, R.W., \& Kashy, D.A. (2011). What does the narcissistic personality inventory really measure? Assessment, 18, 67-87. doi:10.1177/1073191110382845

Ames, D.R., Rose, P., \& Anderson, C.P. (2006). The NPI-16 as a short measure of narcissism. Journal of Research in Personality, 40, 440-450. doi:10.1016/j.jrp.2005.03.002

Bieda, A, Hirschfeld, G., Schonfeld, P., Brailovskaia, J., Zhang, X.C., \& Margraf, J. (2016). Universal happiness? Cross-cultural measurement invariance of scales assessing positive mental health. Psychological Assessment, 29, 408-421. doi:10.1037/pas0000353

Brailovskaia, J., \& Bierhoff, H-W. (2016). Cross-cultural narcissism on Facebook: Relationship between self-presentation, social interaction and the open and covert narcissism on a social networking site in Germany and Russia. Computers in Human Behavior, 55, 251-257. doi:10.1016/j.chb.2015.09.018

Brailovskaia, J., Bierhoff, H.W., \& Margraf, J. (2017). How to identify narcissism with 13 items? Validation of the German Narcissistic Personality Inventory-13 (G-NPI-13). Assessment. doi:10.1177/1073191117740625

Brislin, R.W. (1986). The wording and translation of research instruments. In W.J. Lonneri \& J.W. Berry (Eds.), Cross-cultural research methodology series: Field methods in crosscultural research (pp. 137-164). Beverly Hills, CA: Sage.

Campbell, K. W., Bonacci, A. M., Shelton, J., Exline, J. J., \& Bushman, B. J. (2004). 


\section{CROSS-CULTURAL GENERALISABITY OF THE NPI}

Psychological entitlement: Interpersonal consequences and validation of self-report measure. Journal of Personality Assessment, 83, 29-45.

Chen, F.F. (2007). Sensitivity of goodness of fit indexes to lack of measurement invariance. Structural Equation Modeling, 14, 464-504. doi:10.1080/10705510701301834

Cozma, I., Javadian, G., Gupta, V.K., \& Canever, M. (2014). Narcissistic Personality Inventory. An assessment of measurement equivalence across countries and gender. The International Journal of Management and Business, 5, 105-128.

Cross, S.E., Bacon, P.L., \& Morris, M.L. (2000). The relational-interdependent self-construal and relationships. Journal of Personality and Social Psychology, 78, 791-808. doi:10.1037/022-3514.78.4.791

Fukunishi, I., Nakagawa, T., Nakamura, H., Li, K., Hua, Z.Q., \& Krantz, T.S. (1996). Relationships between type A behavior, narcissism, and maternal closeness for college students of Japan, the United States of America, and the People of the Republic of China. Psychological Reports, 78, 939-944. doi:10.2466/pr0.1996.78.3.939

Gebauer, J.E., Sedikides, C., Verplanken, B., \& Maio, G.R. (2012). Communal narcissism. Journal of Personality and Social Psychology, 103, 854-878. doi:10.1037/a0029629

Gentile, B., Miller, J.D., Hoffman, B.J., Reidy, D.E., Zeichner, A., \& Campbell, W.K. (2013). A test of two brief measures of grandiose narcissism: The Narcissistic Personality Inventory-13 and the Narcissistic Personality Inventory-16. Psychological Assessment, 25, 1120-1136. doi:10.1037/a0033192

Grijalva, E., Newman, D.A., Tay, L., Donnellan, M.B., Harms, P.D., Robins, R.W., \& Yan, T. (2015). Gender differences in narcissism: A meta-analytic review. Psychologial Bulletin, 141, 261-310. doi:10.1037/a0038231 
CROSS-CULTURAL GENERALISABITY OF THE NPI

Hashimoto, H., \& Yamagishi. T. (2013). Two faces of interdependence: Harmony seeking and rejection avoidance. Asian Journal of Social Psychology, 16, 142-151. doi:10.1111/ajsp.12022

Hitokoto, H., \& Uchida, Y. (2015). Interdependent happiness: theoretical importance and measurement validity. Journal of Happiness Studies, 16, 211-239. doi:10.1007/s10902014-9505-8

Hofstede, G., Hofstede, G.J., \& Minkov, M. (2010). Cultures and organizations: software of the mind (rev. 3rd ed.). New York, NY: McGraw-Hill.

Meisel, M.K., Ning, H., Campbell, W.K., \& Goodie, A.S. (2016). Narcissism, overconfidence, and risk taking in U.S. and Chinese students samples. Journal of CrossCultural Psychology, online first. doi:10.1177/0022022115621968

Meredith, W. (1993). Measurement invariance, factor analysis and factorial invariance. Psychometrika, 58, 525-543. doi:10.1007/BF02294825

Miller, J.D., \& Campbell, W.K. (2008). Comparing clinical and social-personality approaches to narcissism. Journal of Personality, 76, 449-476. doi:10.1111/j.14676494.2008.00492.x

Muthén, L.K., \& Muthén, B.O. (2014). Mplus user's guide, 7th ed. Los Angeles, CA: Muthén \& Muthén.

Noguchi, K. (2007). Examination of the content of individualism/collectivism scales in cultural comparisons of the USA and Japan. Asian Journal of Social Psychology, 10, 131-144. doi:10.1111/j.1467-839X.2007.00220.x

Raskin, R., \& Terry, H. (1988). A principal-components analysis of the Narcissistic Personality Inventory and further evidence of its construct validity. Journal of Personality and Social Psychology, 54, 890-902. doi:10.1037/0022-3514.54.5.890 
CROSS-CULTURAL GENERALISABITY OF THE NPI

Rosenberg, M. (1965). Society and the adolescent self-image. Princeton, NJ: Princeton University Press.

Singelis, T.M. (1994). The measurement of independent and interdependent self-construals. Personality and Social Psychology Bulletin, 20, 580-591. doi:10.1177/0146167294205014

Uchida, Y., \& Kitayama, S. (2009). Happiness and unhappiness in east and west: themes and variations. Emotion, 9, 441-456. doi:10.1037/a0015634

Yamaguchi, S., Greenwald, A.G., Banaji, M.R., Murakami, F., Chen, D., Shiomura, K., Kobayashi, C., Cai, H., \& Krendl, A. (2007). Apparent universality of positive implicite self-esteem. Psychological Science, 18, 498-500. doi:10.1111/j.14679280.2007.01928.x 
CROSS-CULTURAL GENERALISABITY OF THE NPI

Table 1

Descriptive statistics and reliability of applied measures across Japan, Poland, and United Kingdom

\begin{tabular}{|c|c|c|c|c|c|}
\hline $\operatorname{Japan}(N=179)$ & $M$ & $S D$ & Min & Max & $\alpha$ \\
\hline Narcissism & 0.28 & 0.19 & 0.00 & 0.77 & .67 \\
\hline Self-esteem & 2.83 & 0.74 & 1.10 & 4.90 & .84 \\
\hline Psychological entitlement & 3.08 & 1.01 & 1.00 & 6.00 & .86 \\
\hline Communal narcissism & 2.81 & 1.13 & 1.00 & 7.00 & .95 \\
\hline Interdependent happiness & 3.39 & 0.68 & 1.67 & 4.89 & .85 \\
\hline Relational-independent self-construal & 3.73 & 0.61 & 1.36 & 5.18 & .85 \\
\hline Poland $(N=298)$ & $M$ & $S D$ & Min & Max & $\alpha$ \\
\hline Narcissism & 0.35 & 0.19 & 0.08 & 0.92 & .67 \\
\hline Self-esteem & 3.31 & 0.74 & 1.00 & 5.00 & .90 \\
\hline Psychological entitlement & 4.14 & 0.97 & 1.00 & 6.67 & .86 \\
\hline Communal narcissism & 3.90 & 0.93 & 1.00 & 7.00 & .91 \\
\hline Interdependent happiness & 3.21 & 0.74 & 1.00 & 5.00 & .86 \\
\hline Relational-independent self-construal & 4.50 & 0.81 & 2.00 & 6.82 & .86 \\
\hline United Kingdom $(N=187)$ & $M$ & $S D$ & Min & Max & $\alpha$ \\
\hline Narcissism & 0.24 & 0.20 & 0.00 & 1.00 & .72 \\
\hline Self-esteem & 3.57 & 0.90 & 1.31 & 6.00 & .91 \\
\hline Psychological entitlement & 3.11 & 1.08 & 1.00 & 6.67 & .86 \\
\hline Communal narcissism & 3.76 & 0.90 & 1.31 & 6.00 & .90 \\
\hline Interdependent happiness & 3.56 & 0.69 & 1.33 & 4.89 & .86 \\
\hline Relational-independent self-construal & 4.05 & 0.68 & 1.73 & 5.36 & .88 \\
\hline
\end{tabular}

Note. $M=$ Mean; $S D=$ Standard Deviation; Min = Minimum; Max = Maximum. 
CROSS-CULTURAL GENERALISABITY OF THE NPI

Table 2

Fit indices of the three-factor model of narcissism in Japan, Poland, and United Kingdom

\begin{tabular}{lcccccc}
\hline Country & $\chi_{(\mathrm{df})}^{2}$ & $p$ & CFI & RMSEA & $90 \%$ CI & $p$ \\
\hline Japan & $78.94_{(62)}$ & .072 & .945 & .039 & $.000-.063$ & .754 \\
Poland & $85.39_{(62)}$ & .026 & .956 & .036 & $.013-.053$ & .910 \\
UK & $94.13_{(62)}$ & .005 & .917 & .053 & $.029-.073$ & .400 \\
\hline
\end{tabular}

Note . CFI = Comparative Fit Index; RMSEA = Root Mean Square of Approximation; $\mathrm{CI}=$ Confidence Interval. 


\section{CROSS-CULTURAL GENERALISABITY OF THE NPI}

\section{Table 3}

Differences in specific items distribution between Polish, Japanese and United Kingdom populations

NPI-13 items (Scale) $\quad \chi^{2}$ (Poland vs $\quad \chi^{2}$ (Poland vs $\quad \chi^{2}$ (UK vs

Japan) UK) Japan)

\begin{tabular}{llll}
\hline 1. I find it easy to manipulate people (EE) & 2.11 & 0.03 & 2.16
\end{tabular}

2. I know that I am a good person because

everybody keeps telling me so (GE)

$16.99 * * * \quad 2.32 \quad 6.32 *$

3. I like having authority over other people

$15.45^{* * *} \quad 18.49 * * * \quad 0.85$

4. I insist upon getting the respect that is due me (EE)

$24.85^{* * *} \quad 4.07^{*} \quad 38.00^{* * *}$

5. I like to show off my body (GE)

6. I have strong will to power (LA)

7. I expect a great deal from other people (EE)

$5.10^{*}$

$6.86^{* *}$

$19.04^{* * *}$

8. I like to look at my body (GE)

$22.93 * * *$

1.85

$11.02 * * *$

9. People always seem to recognise my

authority (LA)

$\begin{array}{lll}7.11^{* *} & 7.57^{* *} & 0.00\end{array}$

10. I will never be satisfied until I get all that I deserve (EE)

$\begin{array}{lll}4.43 * & 14.92 * * * & 28.69 * * *\end{array}$

11. I will usually show off if I get the chance

(GE)

$\begin{array}{lll}4.76^{*} & 1.80 & 9.80 * *\end{array}$

12. I am a born leader (LA)

13. I like to look at myself in the mirror (GE)

Note. $\mathrm{UK}=$ United Kingdom. $\mathrm{EE}=$ Entitlement Exploitativeness; GE = Grandiose Exhibitionism; LA = Leadership Authority.

${ }^{*} p<.05 ; * * p<.01 ; * * * p<.001$ 


\section{CROSS-CULTURAL GENERALISABITY OF THE NPI}

Table 4

Model fit indices of measurement invariance analysis of two-factor model of narcissism among Poland, Japan, and United Kingdom

\begin{tabular}{lcccccc}
\hline Level of invariance & $\chi^{2}(\mathrm{df})$ & $p$ & CFI & RMSEA & $90 \%$ CI & $p$ \\
\hline Configural & $108.11(78)$ & .014 & .965 & .042 & $.020-.060$ & .759 \\
Scalar & $130.15(88)$ & .002 & .951 & .046 & $.028-.063$ & .620 \\
$\Delta$ & -22.04 & .011 & .014 & -.004 & $-.008-.003$ & .139
\end{tabular}

Note. CFI = Comparative Fit Index; RMSEA = Root Mean Square Error of Approximation; $\mathrm{CI}=$ Confidence Interval. 
CROSS-CULTURAL GENERALISABITY OF THE NPI

Table 5

Comparison of latent mean differences across Polish, Japanese, and British populations

\begin{tabular}{llll}
\hline Compared Dyad & Scale & Latent mean & $p$ \\
& & difference & \\
\hline Poland - Japan & Grandiose Exhibitionism & -0.29 & .391 \\
& Leadership/Authority & -3.28 & .595 \\
& & & .141 \\
\hline Poland - United Kingdom & Grandiose Exhibitionism & 0.11 & .128 \\
& Leadership/Authority & -1.39 & .270 \\
& & & .786 \\
\hline Japan - United Kingdom & Grandiose Exhibitionism & 1.11 & \\
& Leadership/Authority & -0.07 & \\
& & & \\
\hline
\end{tabular}

Note. Mean difference with negative sign indicates higher mean for the first country in dyad. 
CROSS-CULTURAL GENERALISABITY OF THE NPI

Table 6

Zero-order correlations of NPI-9 and NPI-13 with external variables across Japan, Poland and the UK

\begin{tabular}{|c|c|c|c|c|c|}
\hline Japan & SES & PES & $\mathrm{CNI}$ & RISC & IHS \\
\hline NPI-9 & $.30 * * *$ & $.50 * * *$ & $.48 * * *$ & .00 & -.07 \\
\hline NPI-13 & $.20 * *$ & $.55 * * *$ & $.48 * * *$ & -.06 & .03 \\
\hline$Z$ & $3.57 * * *$ & -1.86 & 0.00 & 1.87 & -1.25 \\
\hline \multicolumn{6}{|l|}{ Poland } \\
\hline NPI-9 & $.21 * * *$ & $.40 * * *$ & $.31 * * *$ & -.02 & $.17 * *$ \\
\hline NPI-13 & $.12^{*}$ & $.44 * * *$ & $.27 * * *$ & -.01 & .09 \\
\hline$Z$ & $3.92 * * *$ & -1.90 & 1.80 & -0.43 & $3.47 * * *$ \\
\hline \multicolumn{6}{|l|}{ UK } \\
\hline NPI-9 & $.15^{*}$ & $.39 * * *$ & $.24 * * *$ & -.13 & .02 \\
\hline NPI-13 & .12 & $.45^{* * *}$ & $.26^{* * *}$ & -.14 & -.04 \\
\hline$Z$ & 1.19 & $-2.61 * *$ & -0.81 & -0.40 & $2.35^{*}$ \\
\hline
\end{tabular}

Note. SES = Self-esteem scale; PES = Pathological Entitlement Scale; CNI = Communal Narcissism Inventory; RISC = Relational-independent self-construal scale; IHS = Interdependent Happiness Scale. $Z=$ Fisher's $Z$ test.

${ }^{*} p<.05 ; * * p<.01 ; * * * p<.001$ 